\title{
Randomized Comparison of Two Techniques for Titrating Power During Radiofrequency Ablation of Accessory Pathways
}

\author{
S. ADAM STRICKBERGER, M.D., RAUL WEISS, M.D., \\ BRADLEY P. KNIGHT, M.D., MARWAN BAHU, M.D., FRANK BOGUN, M.D., \\ KAREN BRINKMAN, B.S., MARK HARVEY, M.D., RAJIVA GOYAL, M.D., \\ EMILE DAOUD, M.D., K. CHING MAN, D.O., and FRED MORADY, M.D. \\ From the Division of Cardiology, Department of Internal Medicine, \\ University of Michigan Medical Center, Ann Arbor, Michigan
}

\begin{abstract}
Impedance Versus Temperature Monitoring. Introduction: The purpose of this study was to prospectively compare the value of impedance and temperature monitoring during accessory pathway ablation. Temperature and impedance monitoring can be used during radiofrequency ablation of accessory pathways to titrate power to achieve adequate but not excessive tissue heating.

Methods and Results: One hundred thirty-two patients with a single accessory pathway were randomly assigned to undergo ablation using either impedance monitoring or temperature monitoring. During impedance monitoring, the endpoint for titration of power was a 5- to 10- $\Omega$ decrease in the measured impedance while for temperature monitoring the endpoint was to achieve a temperature of $58^{\circ}$ to $62^{\circ} \mathrm{C}$. Two protocols were used. In protocol 1 (90 patients), impedance monitoring was performed with a nonthermistor catheter and temperature monitoring was performed with a thermistor catheter. In protocol 2 (42 patients), a thermistor catheter was used in all patients. In protocol 1 , the success rate $(93 \%$ vs $93 \% ; P=1.0)$, ablation procedure duration ( $57 \pm 56$ vs $41 \pm 41 \mathrm{~min}$ ), fluoroscopy time (48 \pm 29 vs $41 \pm 23 \mathrm{~min} ; P=0.3$ ), number of applications $(6.2 \pm 4.7 \mathrm{vs} 5.7 \pm 4.6 ; P=0.8)$, and the number of applications associated with coagulum formation $(0.1 \pm 0.3$ vs $0.3 \pm 0.6 ; P=0.1)$ were similar in the two groups. In protocol 2 , as in protocol 1 , there were no differences in the success rate $(91 \%$ vs $95 \% ; P=1.0)$, ablation procedure duration ( $49 \pm 37$ vs $62 \pm 55$ min; $P=0.4$ ), fluoroscopy time (46 \pm 24 vs $49 \pm$ $36 \mathrm{~min} ; \mathrm{P}=0.8$ ), number of applications $(6.8 \pm 7.0$ vs $7.8 \pm 12.1 ; P=0.7)$, or number of applications associated with coagulum formation $(0.3 \pm 0.6$ vs $0.2 \pm 0.7 ; P=0.6)$ between the impedance and temperature monitoring groups.

Conclusion: Temperature and impedance monitoring are equally effective in optimizing the results of accessory pathway ablation. (J Cardiovasc Electrophysiol, Vol. 7, pp. 795-801, September 1996)
\end{abstract}

Wolff-Parkinson-White syndrome, thermistor ablation catheter, thermometry

\section{Introduction}

Tissue heating to approximately $60^{\circ} \mathrm{C}$ is required for successful catheter ablation of accessory pathways using radiofrequency energy. ${ }^{1,2}$ Because

Address for correspondence: S. Adam Strickberger, M.D., University of Michigan Medical Center, 1500 East Medical Center Dr., Ann Arbor, MI 48109-0022. Fax: 313-936-7641.

Manuscript received 12 September 1995; Accepted for publication 22 April 1996. variables such as tissue contact pressure affect the amount of power required to achieve adequate tissue heating, ${ }^{1,3-5}$ applications at a fixed power setting may result in insufficient, sufficient, or excessive heating.

Two techniques can be used to titrate power to attain appropriate tissue heating. These include temperature monitoring ${ }^{1.2}$ and impedance monitoring. ${ }^{6}$ During temperature monitoring, the target temperature for accessory pathway ablation is 
TABLE 1

Clinical Characteristics of Protocol 1 Patients

\begin{tabular}{lcccc}
\hline & All Patients & Impedance Monitoring & Temperature Monitoring & P Value \\
\hline Number & 90 & 45 & 45 & -15 \\
Age (years) & $38 \pm 14$ & $38 \pm 15$ & $22 / 23$ & 0.8 \\
Gender (M/F) & $46 / 44$ & $25 / 20$ & & 0.7 \\
AP Location & 29 & 10 & 22 & 0.1 \\
Right free wall & 53 & 31 & 4 & \\
Left free wall & 8 & 4 & & \\
Posteroseptal & & & & \\
\hline
\end{tabular}

AP $=$ accessory pathway; $\mathrm{f}=$ female; $\mathrm{M}=$ male.

approximately $60^{\circ} \mathrm{C} .1,2$ During impedance monitoring, applications of radiofrequency energy associated with a drop in impedance of 5 to $10 \Omega$ are associated with an electrode-tissue interface temperature of approximately $60^{\circ} \mathrm{C} .6$

The clinical utility of these techniques has not been compared. Therefore, the purpose of this study was to prospectively compare impedance and temperature monitoring during radiofrequency catheter ablation of accessory pathways.

\section{Methods}

\section{Study Design}

A comparison of temperature and impedance monitoring was performed using two methodologies. In protocol 1, a thermistor catheter was used in patients randomized to temperature monitoring, and a standard, nonthermistor ablation catheter was used in patients randomized to impedance monitoring. This protocol reflects clinical practice, in that a thermistor catheter was used only when temperature monitoring was desired; otherwise, a standard nonthermistor catheter was used. However, inherent differences between the two types of catheters could influence the results. Therefore, protocol 2 was also performed. In protocol 2, accessory pathway ablation was always performed with the thermistor catheter. However, the patients were ran- domized to power titration guided by temperature monitoring alone, or by impedance monitoring alone. In patients randomized to impedance monitoring, temperature data were not available to the operators during the ablation procedure. Likewise, impedance data were not available to the operators when temperature monitoring was performed.

\section{Characteristics of Subjects}

One hundred thirty-two consecutive patients with a single accessory pathway referred for catheter ablation were enrolled under a protocol approved by the Committee on Human Research at the University of Michigan. Ninety consecutive patients were assigned to protocol 1 , and 42 consecutive patients were assigned to protocol 2. In protocol 1 , there were 46 men and 44 women (mean age $38 \pm 14$ years $[ \pm$ SD $]$ ). Only two patients had structural heart disease. There were no significant differences in clinical characteristics or accessory pathway location between the patients randomized to impedance and temperature monitoring (Table 1). In protocol 2, there were 28 men and 14 women (mean age $37 \pm 15$ years). No patient had structural heart disease, and there were no significant differences in accessory pathway location or clinical characteristics between patients randomized to impedance monitoring or temperature monitoring (Table 2).

TABLE 2

Clinical Characteristics of Protocol 2 Patients

\begin{tabular}{lcccc}
\hline & All Patients & Impedance Monitoring & Temperature Monitoring & P Value \\
\hline Number & 42 & 21 & 21 & - \\
Age (years) & $37 \pm 15$ & $38 \pm 14$ & $36 \pm 16$ & 0.6 \\
Gender (M/F) & $28 / 14$ & $13 / 8$ & $15 / 6$ & 0.7 \\
AP Location & 8 & 3 & 5 & \\
$\quad$ Right free wall & 28 & 16 & 12 & \\
Left free wall & 6 & 2 & 4 & \\
Posteroseptal & & & & \\
\hline AP = accessory pathway; $\mathrm{f}=$ female; $M=$ male &
\end{tabular}




\section{Electrophysiologic Testing and Catheter Ablation}

Electrophysiologic testing was performed with quadripolar electrode catheters positioned in the high right atrium, across the tricuspid valve to record a His-bundle electrogram, and in the right ventricular apex. Leads $\mathrm{V}_{1}, \mathrm{I}$, II, and III, and the intracardiac electrograms were recorded on a Siemans-Elema (Solna, Sweden) Mingograph 7 recorder. Mapping demonstrated that the accessory pathway was located in the right free wall in 35 patients, the left wall in 71 patients, and the posteroseptal area in 8 patients (Tables 1 and 2).

The techniques used for ablation have been previously described. ${ }^{7-10}$ Ablation of right-sided and septal accessory pathways was accomplished by advancing the ablation catheter up the inferior vena cava and positioning it on the atrial side of the tricuspid annulus. For left-sided accessory pathways, the catheter was inserted into a femoral artery, passed across the aortic valve, and positioned on the ventricular side of the mitral annulus.

Target sites for ablation were selected using previously established criteria. "If the accessory pathway was manifest, mapping was performed during sinus rhythm or atrial pacing. If the accessory pathway was concealed, mapping of retrograde atrial activation was accomplished during orthodromic tachycardia or ventricular pacing. Stability of the target site electrogram was required, with stability being defined as $<10 \%$ variation in the atrial or ventricular electrogram amplitude from beat to beat. $7,10,11$

\section{Ablation Catheters}

Two different ablation catheters were used for protocol 1. A 7-French quadripolar catheter with a 4-mm distal electrode and a steerable shaft was used in patients randomized to impedance monitoring (Mansfield EP, Watertown, MA, USA). A 7-French quadripolar electrode catheter with a deflectable shaft in which a thermistor bead was incorporated into the 4-mm distal electrode (EP Technologies, Inc., Sunnyvale, CA, USA) was used in patients randomized to temperature monitoring. The thermistor bead was exposed to the surface and thermally insulated from the surrounding platinum electrode by a polyamide plastic sleeve. Each catheter was individually calibrated and accurate within $2^{\circ}$ from $37^{\circ}$ to $100^{\circ} \mathrm{C}$. A previous study demonstrated that a thermistor isolated from the radiofrequency energy delivery electrode and in contact with the endocardium accurately measures the temperature at the electrode-tissue interface. ${ }^{12}$
The thermistor catheter used in this study was evaluated clinically in a prior study and found to be effective and accurate. ${ }^{1}$ The impedance change measurement was accurate to within $\pm 1 \Omega$ for both the nonthermistor and thermistor catheters.

Only the thermistor ablation catheter (EP Technologies) was used in protocol 2. Patients in protocol 2 were randomly assigned to radiofrequency catheter ablation with temperature monitoring alone, or with impedance monitoring alone. In patients randomly assigned to temperature monitoring, only the temperature was displayed to the operators of the procedure. Likewise, the temperature data from patients randomly assigned to impedance monitoring were not displayed to the operators of the procedure.

\section{Study Protocol}

Radiofrequency energy was delivered by a generator that delivered a continuous, unmodulated sine wave output at $500 \mathrm{kHz}$, and had a maximum output of $50 \mathrm{~W}$ (EP Technologies). The radiofrequency generator required manual modulation of power. This device was interfaced with a personal computer (Toshiba Electronics, Inc.), which continuously measured and recorded power, impedance, and, when available, tip temperature, during each radiofrequency application. Radiofrequency energy was delivered between the 4-mm distal electrode of the ablation catheter and a large adhesive electrode positioned over the left scapula.

In protocols 1 and 2, patients were randomly assigned to temperature monitoring or impedance monitoring. In patients randomly assigned to temperature monitoring, only the temperature was displayed to the operators. The impedance data were recorded and stored for future analysis. The endpoint for temperature monitoring was manual power modulation to achieve a measured temperature of $58^{\circ}$ to $62^{\circ} \mathrm{C}$ at each target site. The power was titrated until either the temperature endpoint was attained or the maximum energy output was delivered $(50 \mathrm{~W})$. If the temperature endpoint was not achieved with maximum power, then the application was terminated. Previous studies in humans have demonstrated $60^{\circ} \mathrm{C}$ to be the average effective temperature for $a b$ lation of accessory pathways. ${ }^{1.2}$

In the patients randomly assigned to undergo impedance monitoring in protocols 1 and 2, only the impedance was measured and displayed. In the impedance monitoring group, the endpoint for manual titration of power was a 5- to $10-\Omega$ decrease in the measured impedance, which corresponds to 
a temperature of approximately $57^{\circ} \mathrm{C} .^{6}$ In both protocols, the power was titrated until the impedance change endpoint was achieved or until the maximum power $(50 \mathrm{~W})$ was delivered. If the endpoint was not achieved with maximum power, then the application was terminated. In protocol 2, impedance monitoring was performed with the thermistor catheter. However, only the impedance measurements and not the temperature measurements were displayed to the operators. The temperature data were recorded and stored for future analysis. In a prior study, coagulum formation was never associated with a $<10-\Omega$ decrement in measured impedance, but was observed in $10 \%$ of applications associated with a $>10-\Omega$ decrement. ${ }^{6}$ Preliminary results from another study were similar. ${ }^{13}$ Therefore, for patients randomized to impedance monitoring, the power at each target site was manually regulated to achieve an impedance decrement of 5 to $10 \Omega$ from the initial impedance value for each application.

The initial power setting was 10 to $25 \mathrm{~W}$ irrespective of the randomization. Titration of power to achieve the appropriate endpoint was continued throughout the energy application, even if loss of accessory pathway conduction was observed before the endpoint was reached. Applications of radiofrequency energy were continued for at least 10 seconds after the target temperature or target impedance was reached. If interruption of accessory pathway conduction was observed, the energy was applied for a total of 30 to 60 seconds.

Success of the procedure, procedure duration, fluoroscopy time, number of radiofrequency applications, and number of applications of energy associated with coagulum formation were recorded for each patient. A successful procedure was defined as complete elimination of accessory pathway conduction. The ablation procedure duration was defined as the time required to perform the procedure after the diagnosis was established and until the final application of radiofrequency energy. If accessory pathway conduction was present after 15 applications of energy, an alternate catheter could be selected. If a catheter type different from that assigned was used, the procedure was considered unsuccessful.

\section{Statistical Analysis}

Continuous variables are expressed as mean \pm 1 SD. Nominal variables were compared by Chisquare analysis, and continuous variables were compared using a $t$-test for unpaired variables or a Student's $t$-test for paired variables, as appropriate. The relationship between continuous variables was assessed with regression analysis. Probability values $<0.05$ were considered statistically significant.

\section{Results}

\section{Protocol 1}

The success rate of ablation was $93 \%$ in patients assigned to impedance monitoring, which was not statistically different from the $93 \%$ success rate in patients assigned to temperature monitoring (Table 3; $\mathrm{P}=1.0$ ). After crossover, the procedure was eventually successful in each patient. The mean procedure duration $(57 \pm 56$ vs 41 $\pm 41 \mathrm{~min} ; \mathrm{P}=0.8$ ) and fluoroscopy time (48 \pm 29 vs $41 \pm 23 \mathrm{~min} ; \mathrm{P}=0.3$ ) in patients randomized to impedance and temperature monitoring, respectively, were not statistically different. The mean number of radiofrequency applications in patients undergoing impedance monitoring was $6.2 \pm 4.7$, compared to $5.7 \pm 4.6$ in patients randomized to impedance monitoring $(\mathrm{P}=0.8)$. There was also no difference in the mean number of applications associated with coagulum formation between the impedance monitoring group $(0.1 \pm 0.3)$ and the temperature monitoring group $(0.3 \pm 0.6 ; \mathrm{P}=0.1)$.

There was no significant difference in success rate, procedure duration, fluoroscopic time, number of applications of energy, or frequency of coagulum formation for patients with right-sided, leftsided, or posteroseptal accessory pathways who were randomized to impedance monitoring or temperature monitoring.

For patients assigned to temperature monitoring, the mean steady-state temperature of all applications was $56^{\circ} \pm 12^{\circ} \mathrm{C}$, while the mean steady-

TABLE 3

Results of Catheter Ablation Guided by

Impedance or Temperature Monitoring: Protocol 1

\begin{tabular}{lccc}
\hline & $\begin{array}{c}\text { Impedance } \\
\text { Monitoring }\end{array}$ & $\begin{array}{c}\text { Temperature } \\
\text { Monitoring }\end{array}$ & P Value \\
\hline $\begin{array}{l}\text { Number of patients } \\
\text { Success rate (\%) }\end{array}$ & 45 & 45 & - \\
$\begin{array}{l}\text { Number of } \\
\text { applications }\end{array}$ & 63 & 93 & 1.0 \\
$\begin{array}{c}\text { Procedure duration } \\
\text { (min) }\end{array}$ & $57 \pm 56$ & $41 \pm 41$ & 0.8 \\
$\begin{array}{c}\text { Fluoroscopy time } \\
\text { (min) }\end{array}$ & $48 \pm 29$ & $41 \pm 23$ & 0.3 \\
$\begin{array}{c}\text { Coagulum } \\
\text { formation/patient }\end{array}$ & $0.1 \pm 0.3$ & $0.3 \pm 0.6$ & 0.1 \\
\hline
\end{tabular}


state temperature for successful applications of energy was $62^{\circ} \pm 10^{\circ} \mathrm{C}$, compared to $55^{\circ} \pm 13^{\circ} \mathrm{C}$ for unsuccessful applications $(\mathrm{P}=0.01)$. The mean change in impedance for all applications was -6.4 $\pm 7.5 \Omega$. Unsuccessful applications of energy were associated with a measured change in impedance of $-6.1 \pm 7.5 \Omega$, while applications associated with elimination of accessory pathway function were associated with an impedance change of $-8.5 \pm$ $4.8 \Omega(P=0.006)$. The change in impedance during successful applications of energy in the impedance and temperature groups were similar $(-9.1$ $\pm 4.5 \Omega$ vs $-7.8 \pm 5.1 \Omega$, respectively; $\mathrm{P}=0.2$ ). A highly significant correlation between the change in impedance and the mean steady-state temperature was observed $(\mathrm{R}=0.6, \mathrm{P}<0.0001)$. Applications of energy associated with coagulum formation were associated with a change in measured impedance of $-10.0 \pm 4.4 \Omega$, while applications without coagulum formation were associated with a change in measured impedance of $-6.2 \pm 7.3 \Omega$ $(P=0.02)$. The mean temperature associated with coagulum formation was $98^{\circ} \pm 18^{\circ} \mathrm{C}$. The mean impedance change observed immediately prior to the coagulum formation was $-13.0 \pm 1.9$ $\Omega$ in the impedance monitoring groups and -12.9 $\pm 2.2 \Omega$ in the temperature monitoring group.

\section{Protocol 2}

In protocol 2, the success rate of ablation was $91 \%$ in patients assigned to impedance monitoring, which did not differ significantly from the 95\% success rate in patients assigned to temperature monitoring (Table $4 ; \mathrm{P}=1.0$ ). The mean procedure duration $(49 \pm 37$ vs $62 \pm 55 \mathrm{~min} ; \mathrm{P}=$ $0.4)$ and fluoroscopic time ( $46 \pm 24$ vs $49 \pm 36$ $\min ; \mathrm{P}=0.8$ ) in patients randomized to impedance monitoring and temperature monitoring, re-

TABLE 4

Results of Catheter Ablation Guicled by Impedance or Temperature Monitoring: Protocol 2

\begin{tabular}{lccc}
\hline & $\begin{array}{c}\text { Impedance } \\
\text { Monitoring }\end{array}$ & $\begin{array}{c}\text { Temperature } \\
\text { Monitoring }\end{array}$ & P Value \\
\hline $\begin{array}{l}\text { Number of patients } \\
\text { Success rate (\%) }\end{array}$ & 21 & 21 & - \\
$\begin{array}{l}\text { Number of } \\
\text { applications }\end{array}$ & $6.8 \pm 7.0$ & $7.8 \pm 12.1$ & 0.7 \\
$\begin{array}{l}\text { Procedure duration } \\
\text { (min) }\end{array}$ & $49 \pm 37$ & $62 \pm 55$ & 0.4 \\
$\begin{array}{l}\text { Fluoroscopy time } \\
\text { (min) }\end{array}$ & $46 \pm 24$ & $49 \pm 36$ & 0.8 \\
$\begin{array}{c}\text { Coagulum } \\
\text { formation/patient }\end{array}$ & $0.3 \pm 0.6$ & $0.2 \pm 0.7$ & 0.6 \\
\hline
\end{tabular}

spectively, were not statistically different. The mean number of radiofrequency applications in patients undergoing impedance monitoring was $6.8 \pm 7.0$, compared to $7.8 \pm 12.1$ in patients randomized to temperature monitoring $(P=0.7)$. Additionally, there was no difference in the frequency of applications associated with coagulum formation in the impedance monitoring group $(0.3$ $\pm 0.6)$ and the temperature monitoring group $(0.2$ $\pm 0.7 ; \mathrm{P}=0.6$ )

When the results were analyzed on the basis of accessory pathway location, there were no statistically significant difference in the success rate, procedure duration, fluoroscopic time, number of radiofrequency energy applications, or number of coagulum formation between patients randomized to impedance or temperature monitoring.

The mean steady-state temperature for all applications of radiofrequency energy for patients assigned to protocol 2 was $53^{\circ} \pm 8^{\circ} \mathrm{C}$. The mean steady-state temperature of applications in patients assigned to impedance monitoring $\left(53^{\circ} \pm 8^{\circ} \mathrm{C}\right)$ was not significantly different than in the patients assigned to temperature monitoring $\left(53^{\circ} \pm 8^{\circ} \mathrm{C}\right.$; $\mathrm{P}=0.8$ ). The mean steady-state temperature of successful applications of radiofrequency energy was $57^{\circ} \pm 7^{\circ} \mathrm{C}$ and was significantly greater than the $52^{\circ} \pm 7^{\circ} \mathrm{C}$ for unsuccessful applications of energy ( $\mathrm{P}<0.001)$. The mean steady-state temperature attained during successful applications of radiofrequency energy in the impedance monitored group $\left(57^{\circ} \pm 7^{\circ} \mathrm{C}\right)$ was not significantly different than in the temperature monitored group $\left(57^{\circ} \pm 8^{\circ} \mathrm{C} ; \mathrm{P}=0.8\right)$. Likewise, the mean steady-state unsuccessful temperature was statistically similar between groups $(P=1.0)$. The mean change in impedance was $-4.1 \pm 0.44 \Omega$ for all applications of energy. The mean change in impedance was $-7.8 \pm 2.7 \Omega$ for successful applications of energy and $-3.5 \pm 4.0 \Omega$ for unsuccessful applications of energy $(P<0.01)$. In the impedance monitoring group, the mean change in impedance for successful energy applications was $-8.5 \pm 1.4 \Omega$, and in the temperature monitoring group was $-7.1 \pm 3.4 \Omega(P=0.4)$. The mean impedance change in the impedance monitoring group $(-3.2 \pm 4.4 \Omega)$ was statistically similar to that in the temperature monitoring group $(-4.3 \pm 3.7 \Omega$, $\mathrm{P}=0.1$ ). A significant correlation between the mean change in impedance and the mean steady-state temperature was observed in the impedance monitoring group $(\mathrm{R}=0.5, \mathrm{P}=0.0001)$ and in the temperature monitoring group $(R=0.7$, $\mathrm{P}<0.0001)$. 


\section{Achievement of Titration Endpoints}

A total of 843 applications of energy were applied during ablation of patients assigned to protocols 1 and 2. For all the patients assigned to impedance monitoring, the power titration goal of an impedance change of -5 to $-10 \Omega$ was achieved in $47 \%$ of the applications. Likewise, for the patients assigned to temperature monitoring, the endpoint of manual titration of power to obtain a temperature of $60^{\circ} \mathrm{C}$ was attained in $51 \%$ of applications $(\mathrm{P}=0.3)$.

\section{Discussion}

\section{Main Findings}

Power titration during radiofrequency ablation of accessory pathways guided by temperature or impedance monitoring was associated with a similar success rate, number of applications of radiofrequency energy, incidence of coagulum formation, fluoroscopy duration, and procedure duration. This was true regardless of whether the same catheter or different catheters were used for temperature and impedance monitoring, indicating that differences in the handling characteristics of the catheters did not affect the results of the study. Therefore, the results of this study demonstrate that impedance monitoring and temperature monitoring are equally effective guides for power titration in patients undergoing radiofrequency ablation of an accessory pathway.

\section{Temperature and Impedance Data}

In protocol 2, the mean temperatures observed during all applications of radiofrequency energy were the same between groups, as were the average temperatures during successful and unsuccessful applications. The mean successful temperature of approximately $60^{\circ} \mathrm{C}$ was similar to that already reported. ${ }^{1}$ The mean temperature measured during successful ablation of an accessory pathway, in both protocols, was approximately $10 \%$ higher than during unsuccessful applications of radiofrequency energy, suggesting that unsuccessful applications may be due, at least in part, to inadequate tissue contact and insufficient tissue heating. Despite having a goal for manual power titration, directed either by impedance or temperature monitoring, the endpoints could be attained in only $50 \%$ of energy applications. This result suggests that inadequate tissue contact is frequently re- sponsible for insufficient heating at the electrodetissue interface and that inadequate tissue contact is a common occurrence.

The temperatures associated with successful and unsuccessful applications of energy in the impedance and temperature monitoring groups of protocol 2 were statistically similar. These results validate the utility of impedance monitoring to accurately predict tissue temperatures of $60^{\circ} \mathrm{C}$.

\section{Advantages and Disadvantages of Temperature and Impedance Monitoring}

Both impedance and temperature monitoring are associated with advantages and disadvantages. First, the list price of catheters with temperature monitoring capabilities is approximately $10 \%$ to $30 \%$ more than that of catheters with only impedance monitoring capabilities. In an environment where cost is an issue, impedance monitoring may offer an advantage. Some thermistor catheter designs may increase the diameter of the catheter, and this may be a disadvantage in pediatric patients. At the present time, catheters with multiple ablation electrodes are being developed to make long linear lesions for the catheter-based treatment of atrial fibrillation. ${ }^{14,15}$ Equipping these types of catheters with thermometry capabilities may be technically difficult and expensive. For these types of catheter designs, impedance monitoring may be more feasible and practical. On the other hand, impedance monitoring may not be feasible with some radiofrequency energy generators. Impedance monitoring is difficult to use with a generator that has an analog scale, or that displays impedance values that fluctuate widely from moment to moment.

\section{Coagulum Formation}

A previous multicenter study ${ }^{2}$ reported the results of radiofrequency catheter ablation of the AV junction, AV nodal reentrant tachycardia, and/or an accessory pathway in 270 patients. In that study, a $1.8 \%$ incidence of coagulum formation was reported. The incidence of coagulum formation was greatest with fixed power applications, and the frequency of coagulum formation decreased significantly when a closed loop temperature monitoring system was used. ${ }^{2}$ The incidence of coagulum formation in the present study was somewhat higher than in the study of Calkins et al., perhaps because power was titrated manually.

In the present study, coagulum formation at times occurred with temperature and impedance moni- 
toring, despite the fact that both provide an assessment of tissue heating. 1,26.13 Two explanations are possible. The first is that manual titration of power may result in coagulum formation because of slow operator responsiveness. The second is that each of these techniques may sometimes fail to predict coagulum formation. Both explanations likely play a role. For instance, Calkins et al. demonstrated that even with closed loop temperature monitoring, coagulum formation was more likely to occur when temperatures $>70^{\circ} \mathrm{C}$ were targeted. Therefore, targeting temperatures of approximately $60^{\circ} \mathrm{C}$ with a closed loop temperature monitoring system may further decrease the frequency of coagulum formation.

\section{Limitations}

All of the patients in this study had an accessory pathway. Therefore, it is unclear whether the results apply to ablation of other arrhythmias such as AV nodal reentrant tachycardia or ventricular tachycardia is not established.

\section{Clinical Implications}

Each of these techniques (impedance and temperature monitoring) has advantages. To use either technique effectively one needs adequate experience. As the results of the present study demonstrate, these two techniques are comparable and independent of the particular catheters used. Because the handling characteristics of catheters with and without thermistors may be different, familiarity with both techniques is important to maximize the results of radiofrequency catheter ablation of accessory pathways.

Acknowledgments: The authors thank the staff of the Clinical Electrophysiology Laboratory for their technical assistance, and Allyson Navyac for her excellent secretarial support.

\section{References}

1. Langberg JJ, Calkins H, El-Atassi R, et al: Temperature monitoring during radiofrequency catheter ablation of accessory pathways. Circulation 1992;86: 1469-1474.

2. Calkins H, Prystowsky E, Carlson M, et al, The Atakr Multicenter Investigators Group: Temperature moni- toring during radiofrequency catheter ablation procedures using closed loop control. Circulation 1994;90: 1279-1286.

3. Hoyt RH, Huang SK, Marcus FI, et al: Factor influencing catheter radiofrequency ablation of the myocardium. J Appl Cardiol 1986; 1:469-486.

4. Haines DE: Determinants of lesion size during radiofrequency catheter ablation: The role of electrodetissue contact pressure and duration of energy delivery. J Cardiovasc Electrophysiol 1991;2:509-515.

5. Haverkamp W, Hindricks G, Gulker H, et al: Coagulation of ventricular myocardium using radiofrequency alternating current: Biophysical aspects and experimental findings. PACE 1989;12:187-195.

6. Strickberger SA, Ravi S, Daoud E, et al: Relation between impedance and temperature during radiofrequency ablation of accessory pathways. Am Heart $\mathbf{J}$ 1995;130:1026-1030.

7. Calkins H, Sousa J, El-Atassi R, et al: Diagnosis and cure of Wolff-Parkinson-White syndrome or paroxysmal supraventricular tachycardia during a single electrophysiologic test. N Engl J Med 1991;324:16121618.

8. Jackman WM, Wang X, Friday KJ, et al: Catheter ablation of accessory atrioventricular pathways (WolffParkinson-White syndrome) by radiofrequency current. N Engl J Med 1991;324:1605-1611.

9. Kuck KH, Schlüter M, Geiger M, et al: Radiofrequency current catheter ablation of accessory atrioventricular pathways. Lancet 1991;337:1557-1561.

10. Calkins H, Langberg J, Sousa J, et al: Radiofrequency catheter ablation of accessory atrioventricular connections in 250 patients. Circulation 1992;85: 1337-1346.

11. Calkins H, Kim YN, Schmaltz S, et al: Electrogram criteria for identification of appropriate target sites for radiofrequency catheter ablation of accessory atrioventricular connections. Circulation 1992;85: 565-573.

12. Blouin LT, Marcus FI, Lampe L: Assessment of effects of a radiofrequency energy field and thermistor location in an electrode catheter on the accuracy of temperature measurement. PACE 1991;14:807-813.

13. Hoffmann E, Remp T, Gerth A, et al: Does impedance monitoring during radiofrequency catheter ablation reduce the risk of impedance rise? Circulation 1993;88:I165 .

14. Swartz JF, Pellersels G, Silvers J, et al: A catheterbased curative approach to atrial fibrillation in humans. Circulation 1994;90(Pt 2):I-335.

15. Haissaguerre M, Gencel L, Fischer B, et al: Successful catheter ablation of atrial fibrillation. J Cardiovasc Electrophysiol 1994;5:1045-1052. 
This document is a scanned copy of a printed document. No warranty is given about the accuracy of the copy. Users should refer to the original published version of the material. 